\title{
GRB AFTERGLOW
}

\author{
B. Zhang ${ }^{1}$
}

\begin{abstract}
Recent observations of broad-band afterglow of gamma-ray bursts (GRBs) suggest that the standard external shock model cannot account for all the data. The observed "afterglow" of some GRBs includes the contributions from at least three emission components. Theoretically, there are naturally three emission sites. Besides the traditional external forward shock, the existence of X-ray flares demands late central engine activities of the GRB central engine, so that an "internal dissipation" region of the late wind is another emission site. After dissipation, this late wind would inevitably catch up with the blastwave, giving rise to a third emission site at a long-lasting reverse shock. Some recent efforts in understanding the broadband afterglow are reviewed.
\end{abstract}

\section{Introduction}

The sub-title of the "Fall 2012 Gamma-Ray Burst Symposium" is "15 years of Gamma-Ray Burst afterglow". It is delightful to recall that afterglow was actually predicted before the discovery. Shortly after the publication of the seminal paper of Mészáros \& Rees (1997), the first X-ray and optical afterglows were discovered for GRB 970228 (Costa et al. 1997; van Paradjis 1997), and the first radio afterglow was discovered for GRB 970508 (Frail et al. 1997). Since then, regular follow-up observations of GRBs have been carried out, and a large amount of broad-band afterglow data have been collected. The launch of the NASA's dedicated GRB mission Swift (Gehrels et al. 2004) opened a new temporal window to study GRB afterglow in the early phase (starting from $\sim 1$ min after the $\gamma$-ray trigger). The launch of the high energy mission Fermi has led to the discovery of an extended $\mathrm{GeV}$ afterglow emission for some bright GRBs (e.g. Ghisellini et al. 2010; Zhang et al. 2011). The observed broad-band data present a perplexing picture, which calls for a rethinking of the origin of GRB afterglow.

${ }^{1}$ Department of Physics and Astronomy, University of Nevada, Las Vegas, USA 


\section{Observation overview}

Before Swift, afterglow observations usually started several hours after the trigger. The optical afterglow at this stage typically displays a power law decay (e.g. Wijers et al. 1997; Harrison et al. 1999), with a steepening "jet" break in the day time scale (Frail et al. 2001). These are consistent with the predictions of the standard external forward shock afterglow models (e.g. Mészáros \& Rees 1997; Sari et al. 1998, 1999; Dai \& Lu 1998; Chevalier \& Li 2000). Irregular wiggles and bumps were observed in some bright GRB optical afterglow lightcurves (e.g. Holland et al. 2003; Lipkin et al. 2004). Some early optical flashes were observed (e.g. Akerlof et al. 1999; Fox et al. 2003), which showed a steep decay slope early on (with a decay index $\sim-2$ ) before flattening to a more normal decay with a decay index -1 . This early component is consistent with being emission from the reverse shock (Mészáros \& Rees 1997, 1999; Sari \& Piran 1999a, 1999b; Kobayashi 2000; Zhang et al. 2003). The rapid slew of XRT and UVOT towards the GRB source allows detections of the GRB early afterglow within less than $100 \mathrm{~s}$ after the trigger. As a result, Swift has provided a rich trove of early afterglow data which revealed many, usually unexpected, interesting features (e.g. Nousek et al. 2006; O'Brien et al. 2006). Zhang et al. (2006) characterized the X-ray afterglow into 5 components: I. a steep decay phase that is smoothly connected with the tail of prompt emission; II. a shallow decay phase (or plateau); III. a "normal" decay phase with decay slope $\sim-1$ as expected in the standard model; IV. a late steepening phase that is consistent with the post-jet-break phase; and V. erratic X-ray flares that overlap on top of the broken power law segments.

It was soon realized that the steep decay phase (Tagliaferri et al. 2005; Zhang et al. 2007) is the tail of prompt emission (Barthelmy et al. 2005). It is likely the high latitude emission after the prompt emission ceases abruptly (Kumar \& Panaitescu 2000; Zhang et al. 2006). This also suggests that the prompt emission region and the afterglow region are detached, establishing the internal origin of the GRB prompt emission (Zhang et al. 2006). The erratic X-ray flares (Burrows et al. 2005) have rapid rising and decaying slopes, which cannot be interpreted within the external shock model. This established the internal origin of the X-ray flares, which calls for delayed central engine activity (Burrows et al. 2005; Zhang et al. 2006; Fan \& Wei 2005; Chincarini et al. 2007; Lazzati \& Perna 2007; Maxham \& Zhang 2009).

The broken power law segments (components II, III and IV defined in Zhang et al. 2006) can be interpreted within the framework of the external shock model, with the phase II interpreted as a refreshed forward shock due to continuous energy injection, phase III interpreted as the normal phase after energy injection is over, and phase IV interpreted as the post-jet-break phase (Zhang et al. 2006; Nousek et al. 2006; Panaitescu et al. 2006). Data analyses (Liang et al. 2007, 2008) suggest that there is essentially no spectral evolution across the two temporal breaks connecting the three segments, and the segment III usually satisfies the $\alpha-\beta$ "closure relations" predicted by the forward shock models (where $\alpha$ and $\beta$ 
are temporal decay index and spectral index of the X-ray afterglow, respectively, with the convention $F_{\nu} \propto t^{-\alpha} \nu^{-\beta}$ ).

Further confusions were introduced by two surprising observations. First, in some cases, an X-ray plateau is found to be followed by a very steep decay, a signature of internal origin of the emission (Troja et al. 2007; Liang et al. 2007; Lyons et al. 2010). This suggests that the late central engine activity of a GRB central engine not only can power erratic flare-like activities, but can also power a steady wind. This may point towards a spinning-down central engine such as a millisecond magnetar (Troja et al. 2007; Lyons et al. 2010). The second puzzling fact is that in some cases, the optical and X-ray lightcurves show a "chromatic" behavior, namely, there is no optical break at the X-ray break time, and vice versa (Panaitescu et al. 2006b; Liang et al. 2007,2008). Since there is no spectral change across the X-ray break (Liang et al. 2007, 2008), this essentially rules out the possibility of interpreting both X-ray and optical data with one single jet component within the standard forward shock model. This drove a wave of modeling invoking emission not from the forward shock. One scenario interprets the entire X-ray and optical afterglow as emission from a long-lasting reverse shock (Uhm \& Beloborodov 2007; Genet et al. 2007), which requires strong suppression of the forward shock emission that would otherwise outshines the reverse shock emission. The other proposal is that the entire X-ray afterglow is due to internal dissipation of a long-lasting central engine wind (Ghisellini et al. 2007; Kumar et al. 2008; Cannizzo \& Gehrels 2009; Yu et al. 2010; Metzger et al. 2011).

To summarize, the X-ray and optical afterglow observations suggest that there might exist probably three emission components that shape the observed afterglow, one erratic X-ray flare component, two power law components - one gives a dominant contribution to the X-ray band and another to the optical band. There are cases that the X-ray and optical lightcurves are "achromatic", so that they are dominated by the same emission component.

Lately, GeV afterglow was observed from several Fermi GRBs. They added more puzzles to the afterglow physics. While in the X-ray band, only a small fraction (say, $5 \%$ ) of afterglow shows a single power law decay from the beginning (most others show the canonical lightcurve with the early steep decay and shallow decay components) (Evans et al. 2009; Liang et al. 2009), all the GeV afterglows show a single power law decay from the beginning (Abdo et al. 2009; Ghisellini et al. 2010; Zhang et al. 2011). Is this due to a selection effect, namely, only those X-ray afterglows with a single power law decay would have a bright enough GeV afterglow? One needs Fermi/LAT-Swift/BAT joint-triggered GRBs to test this idea (so that the early afterglow lightcurves in both X-ray and GeV bands are available). Currently there are two cases, i.e. GRB 090510 (De Pasquale et al. 2010) and GRB 110731A (Ackermann et al. 2013), both showing an achromatic single power law decay in both X-rays and GeV emission. This is consistent with the selection effect interpretation, but more cases are definitely needed to make a robust claim.

It is worth mentioning that even though multiple emission components exist in some GRBs that defy the simplest external shock model, there are indeed a good 
fraction of afterglows that are "well-behaved", namely, they seem to well follow the predictions of the simplest external forward shock model. A systematic analysis is needed to quantify the fraction of GRBs that abide by or defy the simplest models, and to study the possible differences of other observation properties of the two groups.

\section{Standard model and new afterglow paradigm}

The standard afterglow model invokes synchrotron emission from the external forward and (briefly) reverse shocks when the relativistic ejecta launched from the GRB engine interacts with the circumburst medium (Mészáros \& Rees 1997; Sari et al. 1998,1999; Dai \& Lu 1998; Chevalier \& Li 2000). The external shock theory is an elegant theory, since it invokes a limited number of model parameters, and has well predicted spectral and temporal properties. On the other hand, depending on many factors (e.g. energy injection, ambient density profile, collimation of the ejecta, forward vs. reverse shock dynamics, and synchrotron spectral regimes), there is a wide variety of the models. These models have distinct predictions on the afterglow decaying index, the spectral index, and the relation between them (the so-called "closure relation"), which have been widely used to interpret the rich multi-wavelength afterglow observations. Due to the page limit, we cannot discuss these models in detail. The closure relations of several widely used models during the deceleration phase have been collected in Table 1 of Zhang \& Mészáros (2004), and Table 2 of Zhang et al. (2006). A complete thorough review of all the external shock models (including both forward and reverse shocks during the reverse shock crossing stage and self-similar deceleration stage) is being written (Gao et al. 2013).

The puzzling chromatic afterglow data discussed in Section 2 demands at least three emission components to account for the observations. It is encouraging to note that indeed there are three natural emission sites: 1. the traditional external forward shock; 2 . an internal dissipation site in the late wind launched by the central engine; and 3. a long-lasting reverse shock due to the interaction between the late wind and the blastwave. One of the important discoveries by Swift is to realize that the GRB central engines "die hard". The existence of late central engine activities inevitably introduces a messy system with at least these three emission sites. The challenge to understand GRB afterglow lies in identifying the contributions of various components from the data and investigating the relative importance of various emission components with the theoretical models.

\section{Recent progress}

So far there is no investigation that self-consistently solves this messy problem. In the following, I review several recent efforts in our group towards an understanding of the origin of broad-band afterglow. 


\subsection{Internal-external shock model to understand X-ray afterglow}

Maxham \& Zhang (2009) developed a shell model to investigate the interplay between internal shocks and the external shock. The code allows free injections of multiple mini-shells with certain distributions of energy and Lorentz factor. The shells can collide, merge, and emit photons from internal shocks. The leading shell would interact with the ambient medium and form a blast, while other shells can catch up with the blast to boost the total energy of the blast. Even though the details of the colliding processes (forward/reverse shock dynamics) are not introduced, this code can follow the general energy budget distribution in the internal shocks and in the blast, and therefore catches the essence of the internal-external shock model. By allowing multiple injection episodes, one can also reproduce $\mathrm{X}$-ray flares. The general conclusion from such modeling is that X-ray flares demand late reactivation of the central engine. During the epoch with late central engine activity, the blastwave is continuously fed and therefore shows an extended shallow decay phase. One problem of this model is that given the standard forward shock parameters $\left(\epsilon_{e}\right.$ and $\left.\epsilon_{B}\right)$, the afterglow level is relatively bright as compared with prompt emission and X-ray flare emission. This is mostly due to the low energy dissipation efficiency of internal shocks. In order to reproduce the observed $\mathrm{X}$-ray afterglow, one needs to either significantly increase the radiative efficiency of the prompt emission and X-ray flare emission (which demands a high-efficiency magnetic dissipation rather than the conventional internal shock dissipation, e.g. Zhang \& Yan 2011), or to greatly suppress the forward shock emission (by lowering $\epsilon_{e}$ and $\epsilon_{B}$, as is demanded by the reverse-shock dominated models (Uhm \& Beloborodov 2007; Genet et al. 2007).

\subsection{Long-lasting reverse shock model}

In order to investigate the effect of a long-lasting reverse shock in more detail, Uhm et al. (2012) recently carried out a systematic analysis of the forward/reverse shock lightcurves within the framework of a long-lasting reverse shock model that invokes a distribution of Lorentz factor in the ejecta. By applying a Lagrangian description of the blastwave and accurately solving the blastwave dynamics, Uhm et al. (2012) calculated the forward and reverse shock lightcurves for a range of input ejecta stratification profiles. By comparing the light curves from the two shocks, we found that the reverse shock light curves are more sensitive to the input ejecta stratifications, and show richer features in the light curves, including steep declines, plateaus, bumps, re-brightenings, etc.. A collection of ejecta stratification profiles, blastwave dynamics, and the forward/reverse shock lightcurves are presented in Figure 1. In reality, the observed lightcurves should include contributions from the two shocks. The contribution from the reverse shock can show up if the reverse shock emission is enhanced (e.g. due to a higher $\epsilon_{B}$ from a more magnetized ejecta than the medium), or the forward shock emission is suppressed. Further modeling of the superposed light curves is needed in order to confront with the data. In any case, the results open a window to understand the puzzling afterglow data. 

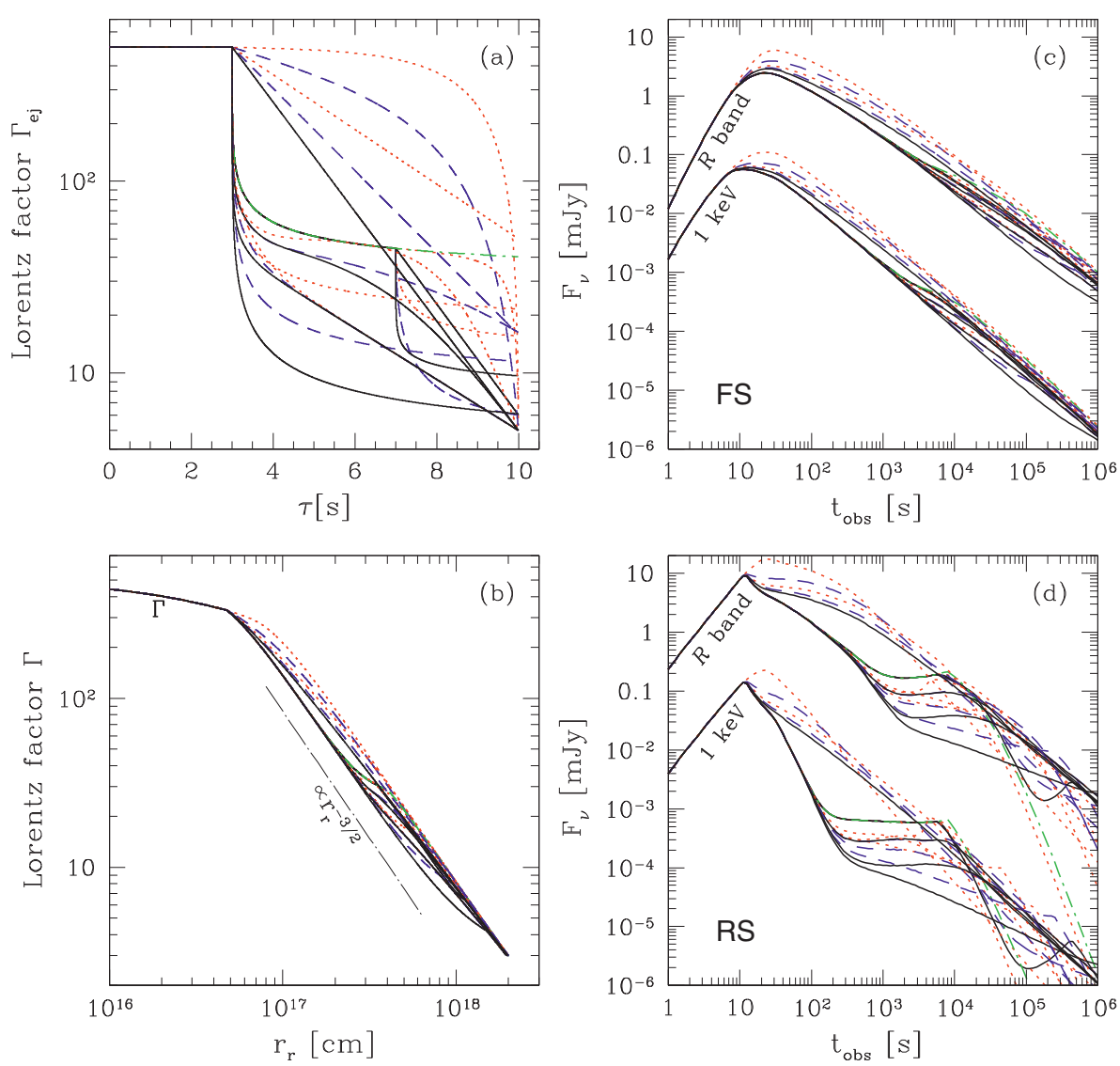

Fig. 1. Blastwave dynamics and forward/reverse shock lightcurves for 20 different models that invoke different ejecta stratification profiles. Top left panel: input ejecta stratification profiles; Bottom left panel: dynamical evolution of the blastwave; Top right panel: forward shock lightcurves in the X-ray and optical bands; Bottom right panel: reverse shock lightcurves in the X-ray and optical bands. From Uhm et al. (2012).

\subsection{Cooling break}

Applying the Lagragian code developed by Lucas Uhm, one finds that the so-called cooling break is extremely smooth. In fact, it takes several decades in energy to approach the predicted $F_{\nu} \propto \nu^{-p / 2}$ regime above the cooling break. Uhm \& Zhang (2013) identified the key physical reason of this smooth transition, which stems from the different cooling histories of electrons accelerated at different epochs. Electrons accelerated earlier underwent a more rapid cooling early on when the magnetic field strength was much higher. This gives an additional spreading of the cooling breaks for different mini-shells. As a result, it is hard to define a global cooling break, so the slow-to-fast cooling transition takes a much longer time. 
This explains the non-detection of any cooling break in 8 years of Swift data. In contrast, the injection frequency $\nu_{m}$ can be reasonably sharp. So whenever a sharp spectral break is observed, it should be related to particle injection rather than cooling. This result applies to a wide range of astrophysical systems involving synchrotron cooling.

\subsection{Origin of $\mathrm{GeV}$ emission}

The single-power-law decay of GeV flux of some GRBs led to the suggestion that the $\mathrm{GeV}$ emission is of the external forward shock origin (Kumar \& Barniol Duran 2009, 2010; Ghisellini et al. 2010). Later studies suggest that during the prompt emission phase (when sub-MeV emission is going on), the main contribution to the $\mathrm{GeV}$ emission is from an internal dissipation region. Maxham et al. (2011) modeled the growth of blastwave during the prompt emission phase using the shell code developed earlier (Maxham \& Zhang 2009), and found that the predicted external shock flux, when scaled to interpret the late GeV afterglow decay, cannot account for the flux during the prompt phase. A similar conclusion was reached independently by He et al. (2011) and Liu \& Wang (2011) using a different approach to model individual GRBs. Observationally, the $\mathrm{GeV}$ peak is found to coincide with one of the spikes of the sub-MeV lightcurve, suggesting its internal origin (Zhang et al. 2011). The identification of the internal origin of $\mathrm{GeV}$ emission during the prompt emission phase is essential to constrain the emission radius through the two-photon pair production opacity constraint, which is found to be much larger than the photosphere radius (Zhang \& Pe'er 2009). This disfavors the photosphere origin of the observed "Band function" spectrum of GRBs, and suggests that the composition of the ejecta is still magnetically dominated in the emission region (Zhang \& Yan 2011).

\section{Summary}

The observations of GRB afterglow have entered a full multi-wavelength era. Even though the standard afterglow model works for some GRBs, a good fraction of GRBs show a chromatic behavior that cannot be interpreted within the standard model. The existence of X-ray flares demand late central engine activities, which inevitably introduces two more emission sites (the internal dissipation site and the reverse shock). This introduces a new paradigm of GRB afterglow, and greatly complicates GRB afterglow modeling.

I thank stimulative collaborations with Z. Lucas Uhm, He Gao, Amanda Maxham, Bin-Bin Zhang, and En-Wei Liang on the topics discussed in this review. This work is partially supported by NSF AST-0908362, NASA NNX10AD48G, and a Cheung Kong Scholar fellowship in Peking University, China.

\section{References}

Abdo, A.A., Ackermann, M., Arimoto, M., et al., 2009, Science, 323, 1688

Ackermann, M., Ajello, M., Asano, K., et al., 2013, ApJ, 763, 71 
Akerlof, C., Balsano, R., Barthelmy, S., et al., 1999, Nature, 398, 400

Barthelmy, S.D., Cannizzo, J.K., Gehrels, N., et al., 2005, ApJ, 635, L133

Burrows, D.N., Romano, P., Falcone, A., et al., 2005, Science, 309, 1833

Cannizzo, J.K., \& Gehrels, N., 2009, ApJ, 700, 1047

Chevalier, R.A., \& Li, Z.-Y., 2000, ApJ, 536, 195

Chincarini, G., Moretti, A., Romano, P., et al., 2007, ApJ, 671, 1903

Costa, E., Frontera, F., Heise, J., et al., 1997, Nature, 387, 783

Dai, Z.G., \& Lu, T., 1998, MNRAS, 298, 87

De Pasquale, M., Schady, P., Kuin, N.P.M., et al., 2010, ApJ, 709, L146

Evans, P.A., Beardmore, A.P., Page, K.L., et al., 2009, MNRAS, 397, 1177

Fan, Y.Z., \& Wei, D.M., 2005, MNRAS, 364, L42

Fox, D.W., Price, P.A., Soderberg, A.M., et al., 2003, ApJ, 586, L5

Frail, D.A., Kulkarni, S.R., Nicastro, L., Feroci, M., \& Taylor, G.B., 1997, Nature, 389, 261

Frail, D.A., Kulkarni, S.R., Sari, R., et al., 2001, ApJ, 562, L55

Gao, H., Lei, W.-H., Zou, Y.-C., Wu, X.-F., \& Zhang, B., 2013, New Astron. Rev., in preparation

Gehrels, N., Chincarini, G., Giommi, P., et al., 2004, ApJ, 611, 1005

Genet, F., Daigne, F., \& Mochkovitch, R., 2007, MNRAS, 381, 732

Ghisellini, G., Ghirlanda, G., Nava, L., \& Celotti, A., 2010, MNRAS, 403, 926

Ghisellini, G., Ghirlanda, G., Nava, L., \& Firmani, C., 2007, ApJ, 658, L75

Harrison, F.A., Bloom, J.S., Frail, D.A., et al., 1999, ApJ, 523, L121

He, H.-N., Wu, X.-F., Toma, K., Wang, X.-Y., \& Mészáros, P., 2011, ApJ, 733, 22

Holland, S.T., Weidinger, M., Fynbo, J.P.U., et al., 2003, AJ, 125, 2291

Kobayashi, S., 2000, ApJ, 545, 807

Kumar, P., \& Barniol Duran, R., 2009, MNRAS, 400, L75

Kumar, P., \& Barniol Duran, R., 2010, MNRAS, 409, 226

Kumar, P., Narayan, R., \& Johnson, J.L., 2008, MNRAS, 750

Kumar, P., \& Panaitescu, A., 2000, ApJ, 541, L51

Lazzati, D., \& Perna, R., 2007, MNRAS, 375, L46

Liang, E.-W., Lü, H.-J., Hou, S.-J., Zhang, B.-B., \& Zhang, B., 2009, ApJ, 707, 328

Liang, E.-W., Racusin, J.L., Zhang, B., Zhang, B.-B., \& Burrows, D.N., 2008, ApJ, 675, 528

Liang, E.-W., Zhang, B.-B., \& Zhang, B., 2007, ApJ, 670, 565

Lipkin, Y.M., Ofek, E.O., Gal-Yam, A., et al., 2004, ApJ, 606, 381

Liu, R.-Y., \& Wang, X.-Y., 2011, ApJ, 730, 1

Lyons, N., O'Brien, P.T., Zhang, B., et al., 2010, MNRAS, 402, 705

Maxham, A., \& Zhang, B., 2009, ApJ, 707, 1623

Maxham, A., Zhang, B.-B., \& Zhang, B., 2011, MNRAS, 415, 77

Mészáros, P., \& Rees, M.J., 1997, ApJ, 476, 232

Mészáros, P., \& Rees, M.J., 1999, MNRAS, 306, L39

Metzger, B.D., Giannios, D., Thompson, T.A., Bucciantini, N., \& Quataert, E., 2011, MNRAS, 413, 2031 
Nousek, J.A., Kouveliotou, C., Grupe, D., et al., 2006, ApJ, 642, 389

O'Brien, P.T., Willingale, R., Osborne, J., et al., 2006, ApJ, 647, 1213

Panaitescu, A., Mészáros, P., Burrows, D., et al., 2006a, MNRAS, 369, 2059

Panaitescu, A., Mészáros, P., Gehrels, N., Burrows, D., \& Nousek, J., 2006b, MNRAS, 366, 1357

Sari, R., \& Piran, T., 1999a, ApJ, 517, L109

Sari, R., \& Piran, T., 1999b, ApJ, 520, 641

Sari, R., Piran, T., \& Halpern, J.P., 1999, ApJ, 519, L17

Sari, R., Piran, T., \& Narayan, R., 1998, ApJ, 497, L17

Tagliaferri, G., Goad, M., Chincarini, G., et al., 2005, Nature, 436, 985

Troja, E., Cusumano, G., O'Brien, P.T., et al., 2007, ApJ, 665, 599

Uhm, Z.L., \& Beloborodov, A.M., 2007, ApJ, 665, L93

Uhm, Z.L., Zhang, B., Hascoët, R., et al., 2012, ApJ, 761, 147

Uhm, Z.L., \& Zhang, B., 2013, ApJ, submitted [arXiv:1301.0291]

van Paradijs, J., Groot, P.J., Galama, T., et al., 1997, Nature, 386, 686

Wijers, R.A.M.J., Rees, M.J., \& Mészáros, P., 1997, MNRAS, 288, L51

Yu, Y.-W., Cheng, K.S., \& Cao, X.-F., 2010, ApJ, 715, 477

Zhang, B., Fan, Y.Z., Dyks, J., et al., 2006, ApJ, 642, 354

Zhang, B., Kobayashi, S., \& Mészáros, P., 2003, ApJ, 595, 950

Zhang, B., \& Mészáros, P., 2004, Int. J. Mod. Phys. A, 19, 2385

Zhang, B., \& Pe'er, A., 2009, ApJ, 700, L65

Zhang, B., \& Yan, H., 2011, ApJ, 726, 90

Zhang, B.-B., Liang, E.-W., \& Zhang, B., 2007, ApJ, 666, 1002

Zhang, B.-B., Zhang, B., Liang, E.-W., et al., 2011, ApJ, 730, 141 
